\title{
EXISTENCE DOMAINS OF HOLOMORPHIC FUNCTIONS OF RESTRICTED GROWTH
}

\author{
M. JARNICKI AND P. PFLUG
}

\begin{abstract}
The paper presents a large class of domains $G$ of holomorphy in $\mathbf{C}^{n}$ such that, for any $N>0$, there exists a nonextendable holomorphic function $f$ on $G$ with $|f| \delta_{G}^{N}$ bounded where $\delta_{G}(z):=\min \left(\left(1+|z|^{2}\right)^{-1 / 2}, \operatorname{dist}(z, \partial G)\right)$. Any fat Reinhardt domain of holomorphy belongs to this class.

On the other hand we characterize those Reinhardt domains of holomorphy which are existence domains of bounded holomorphic functions.
\end{abstract}

1. Introduction. Let $\delta_{0}(z):=\left(1+\|z\|^{2}\right)^{-1 / 2}, z \in \mathbf{C}^{n}$, where \|\| denotes the Euclidean norm in $\mathbf{C}^{n}$. For every open set $G \subset \mathbf{C}^{n}$, put $\delta_{G}:=\min \left\{\rho_{G}, \delta_{0}\right\}$, where $\rho_{G}$ denotes the Euclidean distance to $\mathbf{C}^{n} \backslash G$. For $N \geqslant 0$, let

$$
O^{(N)}\left(G, \delta_{G}\right):=\left\{f \in O(G):\left\|\delta_{G}^{N} \cdot f\right\|_{\infty}<+\infty\right\}
$$

be the space of all holomorphic functions with polynomial growth in $G$ of degree $\leqslant N\left(\|\|_{\infty}\right.$ denotes the supremum norm). Mention that $O^{(0)}\left(G, \delta_{G}\right)$ equals the Banach algebra $H^{\infty}(G)$ of all bounded holomorphic functions in $G$. Set

$$
O^{(0+)}(G):=\bigcap_{N>0} O^{(N)}\left(G, \delta_{G}\right) .
$$

Definition 1.1. $G$ is said to be of type

(a) $H^{\infty}$ (shortly: $G \in H^{\infty}$ ) if $G$ is an $H^{\infty}(G)$-domain of holomorphy;

(b) $O^{(0+)}\left(G \in O^{(0+)}\right)$ if $G$ is an $O^{(0+)}$-domain of holomorphy;

(c) $O^{(>0)}\left(G \in O^{(>0)}\right)$ if, for each $N>0, G$ is an $O^{(N)}\left(G, \delta_{G}\right)$-domain of holomorphy;

(d) $L_{h}^{2}\left(G \in L_{h}^{2}\right)$ if $G$ is an $L_{h}^{2}(G)$-domain of holomorphy, where $L_{h}^{2}(G):=L^{2}(G)$ $\cap O(G) ; L^{2}(G)$ denotes the space of all Lebesgue square integrable functions in $G$.

Let us recall known results.

1.2 [9]. Every domain of holomorphy $G \subset \mathbf{C}^{n}$ is an $O^{(N)}\left(G, \delta_{G}\right)$-domain of holomorphy for $N>2 n$. If $G$ is a bounded domain of holomorphy than the same is true for $N>n$.

$1.3[\mathbf{1 0}]$. Every bounded and fat domain of holomorphy $G \subset \mathbf{C}^{n}$ is of type $L_{h}^{2}$ and, consequently, it is an $O^{(n)}\left(G, \delta_{G}\right)$-domain of holomorphy.

1.4 [12]. Every domain $G \subset \mathbf{C}^{n}$ which is complete w.r.t. the Carathéodory distance $c_{G}$ is of type $H^{\infty}$; here we mean complete in the following sense: For any $c_{G}$-Cauchy-sequence $\left\{z^{\nu}\right\} \subset G$ there is a point $z^{*} \in G$ with $\left\|z^{\nu}-z^{*}\right\| \rightarrow 0$. 
1.5 [11]. Every bounded balanced and $n$-circled domain $G$ of holomorphy is Carathéodory finitely compact (i.e. every $c_{G}$-ball is a relatively compact subset of $G$ ).

1.6 [7]. Let $G$ be a balanced $n$-circled domain of holomorphy. Then, for each $N>0, G$ is the domain of holomorphy with respect to the space $O^{(N)}\left(G, \rho_{G}\right):=$ $\left\{f \in O(G):\left\|\rho_{G}^{N} \cdot f\right\|_{\infty}<+\infty\right\}$.

$1.7[7,14]$. Let $G$ be a fat and $n$-circled domain of holomorphy. Then $G \in H^{\infty}$ iff the space $E(\log G)$ is of rational type-cf. Definitions 3.1 and 3.2. For $n=2$ compare also [1].

Characterizations of balanced domains of type $H^{\infty}$ were given in $[14,15]$. Hartogs domains of type $H^{\infty}$ were studied in $[\mathbf{1 2}, \mathbf{1 3}]$.

1.8 [7]. Let $G$ be as in 1.6. Then $L_{h}^{2}(G) \neq\{0\}$ iff $E(\log G)=\{0\}$.

The problem of continuation of holomorphic functions with polynomial growth across thin sets is considered in the first part of $\S 2$. In the second part we study the problem which open sets of the form $G:=\{z \in D: u(z)<0\}$ are of type $O^{(>0)}$, where $D$ is a domain of holomorphy in $\mathbf{C}^{n}, u \in \operatorname{PSH}(D)$. In particular, we get (cf. 1.6): Let $G$ be an $n$-circled domain of holomorphy. Then $G \in O^{(>0)}$ iff $G$ is fat.

In the case where $G$ is a fat $n$-circled domain of holomorphy, the structures of the spaces $H^{\infty}(G), O^{(0+)}(G)$ and $L_{h}^{2}(G)$ are investigated in $\S 3$. The main results of this section are the following two theorems.

THEOREM (CF. 1.7). Let $G \subset \mathbf{C}^{n}$ be an n-circled domain of holomorphy. Then the following conditions are equivalent:

(i) $G \in H^{\infty}$;

(ii) $G \in O^{(0+)}$;

(iii) $G$ is fat and the space $E(\log G)$ is of rational type.

THEOREM (CF. 1.8). Let $G \subset \mathbf{C}^{n}$ be a fat $n$-circled domain of holomorphy. Then the following conditions are equivalent:

(i) $L_{h}^{2}(G) \neq\{0\}$;

(ii) $E(\log G)=\{0\}$;

(iii) $G \in L_{h}^{2}$.

In $\S 3$, we also present an explicit construction of the $H^{\infty}$-envelope of holomorphy of $G$.

The paper was started during the stay of the first author at the University of Osnabrück, (May 1985) and finished during the stay of the second author at Jagellonian University, Kraków (January-February 1986). We would like to express our gratitude to those universities for their hospitality.

2. Domains of type $O^{(>0)}$. We shall need the following slightly generalized version of the Riemann Removable Singularity Theorem-cf. [5, Chapter I, §C, Theorem 3].

THeOREM 2.1. Let $M$ be a thin subset of a domain $G \subset \mathbf{C}^{n}$ and let $f \in O(G \backslash M)$ satisfy the following condition: for every $a \in G$ there exist a neighborhood $U_{a} \subset G$ and constants $0 \leqslant N_{a}<1, c_{a}>0$ such that

$$
[\operatorname{dist}(z, M)]^{N_{a}} \cdot|f(z)| \leqslant c_{a}, \quad z \in U_{a} \backslash M .
$$

Then $f$ extends holomorphically to $G$. 
The proof is analogous with the proof of the standard version of this theorem. As a direct consequence of Theorem 2.1 we have

Corollary 2.2. Let $M$ be a closed thin subset of a domain $G \subset \mathbf{C}^{n}$. Then, for every $0 \leqslant N<1: O^{(N)}\left(G \backslash M, \delta_{G \backslash M}\right) \subset O(G)$.

REMARK 2.3. In the case $N=0$ we have $H^{\infty}(G \backslash M) \simeq H^{\infty}(G)$. The case $0<N$ $<1$ is different: functions from $O^{(N)}\left(G \backslash M, \delta_{G \backslash M}\right)$ need not be extendable to functions with polynomial growth in $G$. For example:

Let $G:=\{z \in \mathbf{C}: 0<|z|<1\}, f(z):=\exp (1 / z), z \in G$. It is clear that for every $N \geqslant 0, \sup _{G}\left\{\delta_{G}^{N} \cdot|f|\right\}=+\infty$, but one can easily construct a discrete set $M \subset G$ such that $f \in O^{(0+)}(G \backslash M)$.

The following notation will be useful in the sequel because of Theorem 2.1.

Definition 2.4. Let $G$ be an open subset of $\mathbf{C}^{n}$. We denote by $G^{*}$ the set of all points $a \in \mathbf{C}^{n}$ for which there exist an open neighborhood $U_{a}$ and a thin set $M_{a} \subset U_{a}$ such that $U_{a} \backslash M_{a} \subset G$.

From the above definition we immediately obtain

Lemma 2.5. (a) The set $G^{*}$ is open and $G \subset G^{*} \subset \operatorname{int} \bar{G}$.

(b) The set $G^{*} \backslash G$ is thin and

$$
G^{*} \backslash G=\left\{a \in \partial G: \exists U_{a}:(\partial G) \cap U_{a} \text { is thin in } U_{a}\right\} .
$$

(c) $\left(G_{1} \cap G_{2}\right)^{*}=G_{1}^{*} \cap G_{2}^{*}, G_{1}, G_{2} \in$ top $\mathbf{C}^{n}$.

(d) $\left(G_{1} \times G_{2}\right)^{*}=G_{1}^{*} \times G_{2}^{*}, G_{j} \in \operatorname{top} \mathbf{C}^{n_{j}}, j=1,2$.

(e) If $S$ is a connected component of $G$, then $S^{*}$ is a connected component of $G^{*}$.

(f) If $F: \mathbf{C}^{n} \rightarrow \mathbf{C}^{n}$ is biholomorphic, then $F(G)^{*}=F\left(G^{*}\right), G \in$ top $\mathbf{C}^{n}$.

In particular, if $G$ is circled (resp. $n$-circled, balanced, starlike) then $G^{*}$ has the same property.

For $A \subset \mathbf{C}^{n}$, let

$$
\log A:=\left\{x=\left(x_{1}, \ldots, x_{n}\right) \in \mathbf{R}^{n}: e^{x}:=\left(e^{x_{1}}, \ldots, e^{x_{n}}\right) \in A\right\}
$$

be the logarithmic image of $A$.

RemarK 2.6. Let $G$ be an $n$-circled domain. Assume that the set $\log G$ is convex (e.g. $G$ is a domain of holomorphy). Then $\log G=\operatorname{int}(\overline{\log G})=\log \operatorname{int} \bar{G}$, hence (int $\bar{G}) \backslash G \subset\left\{z=\left(z_{1}, \ldots, z_{n}\right) \in \mathbf{C}^{n}: z_{1} \cdots \cdot z_{n}=0\right\}$ and therefore $G^{*}=\operatorname{int} \bar{G}$.

In view of Lemma $2.5(\mathrm{~b})$ and Remark 2.6, Corollary 2.2 implies the following:

COROllary 2.7. For every $0 \leqslant N<1$ :

(a) $O^{(N)}\left(G, \delta_{G}\right) \subset O\left(G^{*}\right)$.

(b) If $G$ is an $O^{(N)}\left(G, \delta_{G}\right)$-domain of holomorphy then $G=G^{*}$.

(c) If $G$ is an $n$-circled $O^{(N)}\left(G, \delta_{G}\right)$-domain of holomorphy then $G$ is fat.

EXAMPLE 2.8. The condition $G=G^{*}$ is only necessary for $G$ to be an $O^{(N)}\left(G, \delta_{G}\right)$-domain of holomorphy with $0 \leqslant N<1$. For example: 
Fix $\frac{1}{2}<r<1$ and let $C_{r}$ denote the generalized Cantor set obtained by removing $r$ 's, i.e.

$$
\begin{aligned}
& C_{r}=\bigcap_{k=1}^{\infty} I^{(k)}, \quad \text { where } I^{(k)}=\bigcup_{j=1}^{2^{k}} I_{j}^{(k)}, \\
& I_{1}^{(1)}=\left[0, \frac{1-r}{2}\right], \quad I_{2}^{(1)}=\left[1-\frac{1-r}{2}, 1\right], \\
& I_{1}^{(2)}=\left[0,\left(\frac{1-r}{2}\right)^{2}\right], \quad I_{2}^{(2)}=\left[\frac{1-r}{2}-\left(\frac{1-r}{2}\right)^{2}, \frac{1-r}{2}\right] . \\
& I_{3}^{(2)}=\left[\frac{1+r}{2}, \frac{1+r}{2}+\left(\frac{1-r}{2}\right)^{2}\right], \quad I_{4}^{(2)}=\left[1-\left(\frac{1-r}{2}\right)^{2}, 1\right], \ldots, \text { etc. }
\end{aligned}
$$

Let $K_{r}:=C_{r} \times C_{r}, G_{r}:=B_{100} \backslash K_{r}$, where $B_{\tau}:=\{z \in \mathbf{C}:|z|<\tau\}, \tau>0$.

It is clear that $\left(G_{r}\right)^{*}=G_{r}$. We shall show that, if

$$
\left(\frac{1-r}{2}\right)^{1-N}<\frac{1}{4}
$$

then $O^{(N)}\left(G_{r}, \delta_{G_{r}}\right) \subset O\left(B_{100}\right)$.

Fix $N$ with (2.1) and let $f \in O^{(N)}\left(G_{r}, \delta_{G_{r}}\right)$. Set

$$
\tilde{f}(z)=\frac{1}{2 \pi i} \int_{\partial B_{80}} \frac{f(\zeta)}{\zeta-z} d \zeta, \quad z \in B_{80} .
$$

It suffices to prove that

$$
\tilde{f}=f \quad \text { in } B_{80} \backslash K_{r} .
$$

Let $x_{j}^{(k)}$ denote the middle point of $I_{j}^{(k)}$,

and let

$$
t_{k}:=\frac{1}{2}\left(\frac{1-r}{2}\right)^{k}+\frac{r}{4}\left(\frac{1-r}{2}\right)^{k-1}
$$

$$
\begin{gathered}
S_{j}^{(k)}:=\left[x_{j}^{(k)}-t_{k}, x_{j}^{(k)}+t_{k}\right], \\
\Delta_{i, j}^{(k)}:=S_{i}^{(k)} \times S_{j}^{(k)}, \quad \Delta^{(k)}:=\bigcup_{i, j=1}^{2^{k}} \Delta_{i, j}^{(k)} .
\end{gathered}
$$

Observe that

$$
\begin{aligned}
& \Delta_{i, j}^{(k)} \cap \Delta_{i^{\prime}, j^{\prime}}^{(k)}=\varnothing, \quad(i, j) \neq\left(i^{\prime}, j^{\prime}\right), \\
& \Delta^{(k+1)} \subset \Delta^{(k)}, \quad K_{r}=\bigcap_{k=1}^{\infty} \Delta^{(k)} .
\end{aligned}
$$

By the Cauchy integral formula we get $f(z)=\tilde{f}(z)+g_{k}(z), z \in B_{80} \backslash \Delta^{(k)}$, where

$$
g_{k}(z):=-\frac{1}{2 \pi i} \sum_{i, j=1}^{2^{k}} \int_{\partial \Delta_{i, j}^{(k)}} \frac{f(\zeta)}{\zeta-z} d \zeta, \quad z \in B_{80} \backslash \Delta^{(k)} .
$$

Fix $\left.z_{0} \in B_{80} \backslash \Delta^{\left(k_{0}\right)}\right)$. For $k \geqslant k_{0}$ we have

$$
\left|g_{k}\left(z_{0}\right)\right| \leqslant \frac{4}{\pi}\left(\frac{4}{r}\right)^{N} \frac{\left\|\delta_{G}^{N} f\right\|_{\infty}}{\operatorname{dist}\left(z_{0}, \Delta^{\left(k_{0}\right)}\right)}\left[4\left(\frac{1-r}{2}\right)^{1-N}\right]^{k-1} \rightarrow 0 \text { as } k \rightarrow+\infty .
$$

Consequently, in view of (2.3), we get (2.2). 
REMARK 2.9. It is known (cf. [4]) that, for $0<r<\frac{1}{2}$, the domain $G_{r}$ is of type $H^{\infty}$. One can prove that $H^{\infty}\left(G_{1 / 2}\right) \simeq H^{\infty}\left(B_{100}\right)$ (cf. [4]). The problem whether $G_{1 / 2} \in$ $O^{(>0)}$ is still open. More generally, one can ask for characterizing the removable singularity sets for $O^{(N)}, O^{(>0)}$ and $O^{(0+)}$.

In view of Corollary 2.7(a), we introduce the following notion.

Definition 2.10. Set

$$
O^{(N)}\left(G^{*}, \delta_{G}\right):=O\left(G^{*}\right) \cap O^{(N)}\left(G, \delta_{G}\right), \quad N \geqslant 0 .
$$

REMARK 2.11. (a) The space $O^{(N)}\left(G^{*}, \delta_{G}\right)$ endowed with the seminorms

$$
O^{(N)}\left(G^{*}, \delta_{G}\right) \ni f \rightarrow\left\|\delta_{G}^{N} f\right\|_{\infty}, \quad O^{(N)}\left(G^{*}, \delta_{G}\right) \ni f \rightarrow \sup _{K}|f|, \quad K \Subset G^{*},
$$

is a complex Fréchet space.

(b) If $0 \leqslant N<1$, then in view of Corollary 2.7(a), by the open mapping theorem, the space $O^{(N)}\left(G^{*}, \delta_{G}\right)$ is algebraically and topologically isomorphic to $O^{(N)}\left(G, \delta_{G}\right)$.

Definition 2.12. We say that $G$ is of type $O^{\left(0^{*}\right)}\left(G \in O^{\left(0^{*}\right)}\right)$, if for each $N>0$, $G^{*}$ is an $O^{(N)}\left(G^{*}, \delta_{G}\right)$-domain of holomorphy.

In the second part of this section we study open sets of the form $G=\{z \in$ $D: u(z)<0\}$, where $D$ is a domain of holomorphy in $\mathbf{C}^{n}, u \in \operatorname{PSH}(D)$.

2.13. Observe that $G^{*} \cap D=G$. In particular, if $D=D^{*}\left(\right.$ e.g. $\left.D \in O^{(>0)}\right)$, then $G=G^{*}$.

Let us consider the following classes of plurisubharmonic functions.

Definition 2.14. For $N \geqslant 0$, let $\mathscr{R}^{(N)}(D)$ denote the set of all functions $u \in$ $\operatorname{PSH}(D)$ such that there exist $\left(f_{\nu}\right)_{\nu=1}^{\infty} \subset O(D),\left(k_{\nu}\right)_{\nu=1}^{\infty} \subset \mathbf{R}_{>0}$ for which

$$
\begin{aligned}
& k_{\nu} \rightarrow 0 \text { as } \nu \rightarrow+\infty ; \\
& \left(\limsup _{\nu \rightarrow+\infty} k_{\nu} \cdot \ln \left|f_{\nu}\right|\right)^{*} \geqslant u \text { in } D,
\end{aligned}
$$

where "*" denotes the upper regularization,

$$
\delta_{G}^{N}\left|f_{\nu}\right| \leqslant 1 \quad \nu \geqslant 1, \quad(G=\{u<0\}, G \text { depends on } u !) .
$$

Set

$$
\mathscr{R}^{(0+)}(D):=\bigcap_{N>0} \mathscr{R}^{(N)}(D) .
$$

Definition 2.15. Let $\mathscr{E}(D)$ denote the set of all functions $u \in \operatorname{PSH}(D)$ for which there exist $\Phi_{0} \in O(D), \Phi_{0} \not \equiv 0, k \in \mathbf{N}, \Phi_{1}, \ldots, \Phi_{k}$-nonzero rational functions holomorphic in $D$ and $\alpha_{0}, \ldots, \alpha_{k}>0$ such that

$$
u=\alpha_{0} \cdot \ln \left|\Phi_{0}\right|+\cdots+\alpha_{k} \cdot \ln \left|\Phi_{k}\right| .
$$

THEOREM 2.16. $\mathscr{E}(D) \subset \mathscr{R}^{(0+)}(D)$.

Proof. Fix $u \in \mathscr{E}(D)$ and let $\Phi_{0}, \ldots, \Phi_{k}, \alpha_{0}, \ldots, \alpha_{k}$ be as in Definition 2.15. We can assume that $\alpha_{0}=1$. Let $P_{1}, \ldots, P_{2 k}$ be complex polynomials such that

$$
\Phi_{j}=P_{j} / P_{k+j}, \quad P_{k+j}(z) \neq 0, \quad z \in D, j=1, \ldots, k .
$$


Let $R:=P_{1} \cdots P_{2 k}, d:=\operatorname{deg} R$. Fix $N>0$ and let $\varepsilon>0$ be such that $3 d \varepsilon \leqslant N$. By the Kronecker theorem [2, Chapter VII, Proposition 2 (see also [3]) there exist sequences $\left(p_{j v}\right)_{\nu=1}^{\infty} \subset \mathbf{N}, j=1, \ldots, k,\left(q_{\nu}\right)_{\nu=1}^{\infty} \subset \mathbf{N}$ such that

$$
\begin{gathered}
q_{\nu} \rightarrow+\infty \quad \text { as } \nu \rightarrow+\infty, \\
\left|p_{j \nu}-\alpha_{j} q_{\nu}\right| \leqslant \varepsilon, \quad \nu \geqslant 1, j=1, \ldots, k .
\end{gathered}
$$

Set

$$
g_{\nu}:=\Phi_{0}^{q_{\nu}} \cdot \Phi_{1}^{p_{1 \nu}} \cdots \Phi_{k}^{p_{k \nu}}, \quad \nu \geqslant 1 .
$$

In view of (2.7) and (2.8) we have

$$
\lim _{\nu \rightarrow+\infty} \frac{1}{q_{\nu}} \cdot \ln \left|g_{\nu}\right|=u \text { in } D .
$$

We shall show that there exists a constant $c>0$ such that

$$
\delta_{G}^{N} \cdot\left|g_{\nu}\right| \leqslant c, \quad \nu \geqslant 1 .
$$

Observe that, if (2.9) holds, then the sequences $\left(g_{\nu} / c\right)_{\nu=1}^{\infty},\left(1 / q_{\nu}\right)_{\nu=1}^{\infty}$ satisfy all the conditions of Definition 2.14 and, consequently, $u \in \mathscr{R}^{(N)}(D)$.

The proof of (2.9) is based on the following lemma.

Lemma 2.17 [8, Proposition 1]. Let $R$ be a nonzero complex polynomial of $n$-complex variables, $d:=\operatorname{deg} R$. Then, for every $\mu>0$, there exists a constant $c(R, \mu)>0$ such that for every $G \in \operatorname{top} \mathbf{C}^{n}$ and for every plurisubharmonic function $\varphi: G \rightarrow \mathbf{R}_{+}:$

$$
\left\|\delta_{G}^{\mu+d} \cdot \varphi\right\|_{\infty} \leqslant c(R, \mu) \cdot\left\|\delta_{G}^{\mu} \cdot R \cdot \varphi\right\|_{\infty} .
$$

For the proof of this lemma see the Appendix.

According to the lemma it is sufficient to show that there exists a constant $c_{0}>0$ such that

$$
\delta_{0}^{2 d} \cdot|R| \cdot\left|H g_{\nu}\right|^{1 / \varepsilon} \leqslant c_{0} \quad \text { in } G, \nu \geqslant 1 .
$$

The set $G \backslash u^{-1}(-\infty)$ is dense in $G$, so it is enough to prove that (2.10) holds true in $G \backslash u^{-1}(-\infty)$.

Fix $a \in G$ with $u(a)>-\infty$ and let

$$
J:=\left\{j \in\{1, \ldots, 2 k\}:\left|P_{j}(a)\right|>1\right\}, \quad I:=\{1, \ldots, 2 k\} \backslash J .
$$

In view of (2.8) one gets

$$
\begin{aligned}
\left|g_{\nu}(a)\right| & \leqslant \frac{\left|g_{\nu}(a)\right|}{\exp \left[q_{\nu} u(a)\right]}=\prod_{j=1}^{k}\left|\Phi_{j}(a)\right|^{p_{j \nu}-\alpha_{j} q_{\nu}} \\
& \leqslant\left|\frac{\prod_{j \in J} P_{j}(a)}{\prod_{i \in I} P_{i}(a)}\right|^{\varepsilon}, \quad \nu \geqslant 1,
\end{aligned}
$$

where $\prod_{i \in \varnothing} \cdots:=1$. Consequently,

$$
\delta_{0}^{2 d}(a) \cdot|R(a)| \cdot\left|g_{\nu}(a)\right|^{1 / \varepsilon} \leqslant\left|\delta_{0}^{d}(a) \cdot \prod_{j \in J} P_{j}(a)\right|^{2} \leqslant\left(c_{1} \cdots c_{k}\right)^{2},
$$

where $c_{j}:=\max \left\{1,\left\|\delta_{0}^{\operatorname{deg} P_{j}} \cdot P_{j}\right\|_{\infty}\right\}, j=1, \ldots, k$. Q.E.D. 
THEOREM 2.18. Let $u \in \mathscr{R}^{(N)}(D)$. Assume that either $D^{*}$ is an $O^{(N)}\left(D^{*}, \delta_{D}\right)$ domain of holomorphy (e.g. $\left.D \in O^{\left(0^{*}\right)}\right)$ or $\bar{G} \subset D^{*}$.

(a) If $G=G^{*}$ then $G$ is an $O^{(N)}\left(G, \delta_{G}\right)$-domain of holomorphy.

(b) If $0 \leqslant N<1$ then $G^{*}$ is an $O^{(N)}\left(G^{*}, \delta_{G}\right)$-domain of holomorphy.

Proof. The assertions (a) and (b) will be proved simultaneously. Assume that either $G=G^{*}$ or $0 \leqslant N<1$ and suppose that $G^{*}$ is not an $O^{(N)}\left(G^{*}, \delta_{G}\right)$-domain of holomorphy. Let $G_{0}, \tilde{G}$ be domains in $\mathrm{C}^{n}$ such that

$$
\varnothing \neq G_{0} \subset G^{*} \cap \tilde{G}, \quad \tilde{G} \not \subset G^{*}, \quad \forall f \in O^{(N)}\left(G^{*}, \delta_{G}\right) \exists \tilde{f} \in O(\tilde{G}): \tilde{f}=f \text { in } G_{0} .
$$

If $D^{*}$ is an $O^{(N)}\left(D^{*}, \delta_{D}\right)$-domain of holomorphy then obviously $\tilde{G} \subset D^{*}$. If $\bar{G} \subset D^{*}$, then, without loss of generality, we may assume that $\tilde{G} \subset D^{*}$. By the open mapping theorem, in view of Remark 2.11(b), for every $a \in \tilde{G}$ there exists $c(a)>0$ such that

$$
|\tilde{f}(a)| \leqslant c(a)\left\|\delta_{G}^{N} f\right\|_{\infty}, \quad f \in O^{(N)}\left(G^{*}, \delta_{G}\right) .
$$

Let $\left(f_{\nu}\right)_{\nu=1}^{\infty},\left(k_{\nu}\right)_{\nu=1}^{\infty}$ be as in Definition 2.14. If $0 \leqslant N<1$, then in view of (2.6) and Corollary 2.7(a), we can assume that $f_{\nu} \in O^{(N)}\left(G^{*}, \delta_{G}\right), \nu \geqslant 1$. By (2.12) we get

$$
\left|\tilde{f}_{\nu}(a)\right| \leqslant c(a), \quad \nu \geqslant 1 .
$$

The set $D^{*} \backslash D$ is thin, hence the set $\tilde{G} \cap D=\tilde{G} \backslash\left(D^{*} \backslash D\right)$ is connected and therefore $\tilde{f}_{\nu}=f_{\nu}$ in $\tilde{G} \cap D, \nu \geqslant 1$. Hence, according to (2.13),

$$
\left|f_{\nu}(a)\right| \leqslant c(a), \quad \nu \geqslant 1, a \in \tilde{G} \cap D .
$$

Then, in view of (2.4) and (2.5): $u \leqslant 0$ in $\tilde{G} \cap D$, which (by the maximum principle) implies that $\tilde{G} \cap D \subset G$. Finally $\tilde{G} \subset G^{*}$ which contradicts (2.11). Q.E.D.

Theorems 2.16 and 2.18 imply the following:

Corollary 2.19. Let $u \in \mathscr{R}^{(0+)}(D)($ e.g. $u \in \mathscr{E}(D))$. Assume that either $D \in$ $O^{\left(0^{*}\right)}$ or $\bar{G} \subset D^{*}$. Then

(a) $G \in O^{\left(0^{*}\right)}$

In particular:

(b) $G \in O^{(>0)}$ iff $G=G^{*}$.

(c) $D \in O^{(>0)} \Rightarrow G \in O^{(>0)}(c f .2 .13)$.

EXAMPLE 2.20. Let $P_{1}, \ldots, P_{2 k}$ be nonzero complex polynomials such that the polynomials $Q:=P_{1} \cdots P_{k}, R:=P_{k+1} \cdots P_{2 k}$ have no common factor and let $\alpha_{1}, \ldots, \alpha_{k}>0$. Define

$$
\begin{aligned}
& V=\{R=0\}, \quad D \in \mathbf{C}^{n} \backslash V \quad\left(\text { note that } D^{*}=\mathbf{C}^{n}\right), \\
& \Phi_{j}=P_{j} / P_{k+j}, \quad j=1, \ldots, k, \quad u=\alpha_{1} \ln \left|\Phi_{1}\right|+\cdots+\alpha_{k} \ln \left|\Phi_{k}\right|, \\
& G=\{z \in D: u(z)<0\} .
\end{aligned}
$$

Then $G=G^{*}$ and so, by Corollary 2.19(b), we have $G \in O^{(>0)}$.

It seems to be interesting that, in some cases, the condition $u \in \mathscr{R}^{(N)}(D)$ is also necessary for $G$ to be an $O^{(N)}\left(G, \delta_{G}\right)$-domain of holomorphy, namely: 
Let $\mathscr{H}\left(\mathbf{C}^{n}\right)$ denote the set of all plurisubharmonic functions $h: \mathbf{C}^{n} \rightarrow \mathbf{R}, h \not \equiv 0$, such that $h(\lambda z)=|\lambda| \cdot h(z), z \in \mathbf{C}^{n}, \lambda \in \mathbf{C}$.

It is known that a domain $G \subsetneq \mathbf{C}^{n}$ is a balanced domain of holomorphy iff there exists $h \in \mathscr{H}\left(\mathbf{C}^{n}\right)$ such that $G=G_{h}:=\{h<1\}$ (cf. $\left.[\mathbf{1 3}, \mathbf{1 4}]\right)$. Note that $\left(G_{h}\right)^{*}=G_{h}$ (cf. 2.13).

Proposition 2.21. Let $h \in \mathscr{H}\left(\mathbf{C}^{n}\right), G:=G_{h}, N \geqslant 0$. Then the following conditions are equivalent:

(i) $G$ is an $O^{(N)}\left(G, \delta_{G}\right)$-domain of holomorphy;

(ii) $\ln h \in \mathscr{R}^{(N)}\left(\mathbf{C}^{n}\right)$ :

(iii) there exists a sequence $\left(Q_{j}\right)_{j=1}^{\infty}$ of homogeneous polynomials such that

$$
\begin{gathered}
\left(\limsup _{j \rightarrow+\infty}\left|Q_{j}\right|^{1 / \operatorname{deg} Q_{j}}\right)^{*}=h \quad \text { in } \mathbf{C}^{n}, \\
\delta_{G}^{N} \cdot\left|Q_{j}\right| \leqslant 1, \quad j \geqslant 1 .
\end{gathered}
$$

In the case $N=0$, the above result reduces to the known characterization of balanced domains of type $H^{\infty}-\mathrm{cf}$. [14, Theorem 3.1].

Proof. The implication (iii) $\Rightarrow$ (ii) is obvious. The implication (ii) $\Rightarrow$ (i) follows from Theorem 2.18. It remains to prove that (i) $\Rightarrow$ (iii). Let $f \in O^{(N)}\left(G, \delta_{G}\right)$ be a nonextendable function with $\left\|\delta_{G}^{N} f\right\|_{\infty} \leqslant 1$. Let $\sum_{j=0}^{\infty} Q_{j}$ denote the expansion of $f$ into the series of homogeneous polynomials. Since $f$ is noncontinuable we get (2.14). In view of the Cauchy inequalities we have

$$
\left|Q_{j}(z)\right| \leqslant \max \left\{\delta_{G}^{-N}(\lambda z):|\lambda|=1\right\}, \quad z \in G, j \geqslant 1 .
$$

Since $G$ is balanced, so $\delta_{G}(\lambda z)=\delta_{G}(z), z \in G,|\lambda|=1$ and hence we have (2.15).

REMARK. It is not known whether every balanced domain of holomorphy is of type $O^{(>0)}$-cf. [15, §5].

Using analogous methods as before one can prove the following result.

Proposition 2.22. Let $v \in \operatorname{PSH}(D)$ and let

$$
\Omega:=\{(z, \omega) \in D \times \mathbf{C}: v(z)+\ln |\omega|<0\} .
$$

Fix $N \geqslant 0$. Assume that $\Omega=\Omega^{*}$ and suppose that either $D^{*}$ is an $O^{(N)}\left(D^{*}, \delta_{D}\right)$ domain of holomorphy of $\bar{\Omega} \subset D^{*} \times \mathbf{C}$. Then the following conditions are equivalent:

(i) $\Omega$ is an $O^{(N)}\left(\Omega, \delta_{\Omega}\right)$-domain of holomorphy;

(ii) $v+\ln |\omega| \in \mathscr{R}^{(N)}(D \times \mathbf{C})$;

(iii) there exists a sequence $\left(F_{j}\right)_{j=1}^{\infty} \subset O(D)$ such that

$$
\begin{aligned}
& \left(\limsup _{j \rightarrow+\infty} \frac{1}{j} \ln \left|F_{j}\right|\right)^{*}=v \quad \text { in } D, \\
& \delta_{\Omega}^{N}(z, \omega)\left|F_{j}(z) \omega^{j}\right| \leqslant 1, \quad(z, \omega) \in \Omega, j \geqslant 1 .
\end{aligned}
$$

Note that in the case $D=\mathbf{C}^{n}, N=0$, the above result generalizes Proposition 2.14 from [13]; compare also [12]. 
REMARK. It would be interesting to know whether Sibony's main example in [12] is of type $O^{(>0)}$ - cf. [7, §2].

Theorem 2.18 and Corollary 2.19 can be easily extended to the following theorem.

Theorem 2.18'. Let $u \in \operatorname{PSH}(D)$ be such that $u=\left(\sup _{i \in I} u_{i}\right)^{*}$, where $\left(u_{i}\right)_{i \in I} \subset$ $\mathscr{R}^{(N)}(D)$. Assume that $D^{*}$ is an $O^{(N)}\left(D^{*}, \delta_{D}\right)$-domain of holomorphy.

(a) If $G=G^{*}$ then $G$ is an $O^{(N)}\left(G, \delta_{G}\right)$-domain of holomorphy.

(b) If $0 \leqslant N<1$ then $G^{*}$ is an $O^{(N)}\left(G^{*}, \delta_{G}\right)$-domain of holomorphy.

COROllaRY 2.19'. Let $u=\left(\sup _{i \in I} u_{i}\right)^{*}$, where $\left(u_{i}\right)_{i \in I} \subset \mathscr{R}^{(0+)}(D)\left(\right.$ e.g. $\left(u_{i}\right)_{i \in I}$ $\subset \mathscr{E}(D))$. Assume that $D \in O^{\left(0^{*}\right)}$. Then

(a) $G \in O^{\left(0^{*}\right)}$;

(b) $G \in O^{(>0)}$ iff $G=G^{*}$;

(c) $D \in O^{(>0)} \Rightarrow G \in O^{(>0)}$.

REMARK 2.23. Let $h \in \mathscr{H}\left(\mathbf{C}^{n}\right)$. Then the following conditions are equivalent:

(i) $\ln h=\left(\sup _{i \in I} u_{i}\right)^{*}$, where $\left(u_{i}\right)_{i \in I} \subset \mathscr{E}\left(\mathbf{C}^{n}\right)$;

(ii) $h=\hat{h}:=\left[\sup \left\{\varphi \in S\left(\mathbf{C}^{n}\right): \varphi \leqslant h\right\}\right]^{*}$,

where $S\left(\mathbf{C}^{n}\right):=\left\{\varphi \in \mathscr{H}\left(\mathbf{C}^{n}\right): \ln \varphi \in \mathscr{E}\left(\mathbf{C}^{n}\right)\right\}$; note that, for a function $\varphi \in \mathscr{H}\left(\mathbf{C}^{n}\right)$, $\ln \varphi \in \mathscr{E}\left(\mathbf{C}^{n}\right)$ if and only if there exists $k \in \mathbf{N}, Q_{1}, \ldots, Q_{k}$-homogeneous polynomials, $\alpha_{1}, \ldots, \alpha_{k}>0$ such that $\alpha_{1} \operatorname{deg} Q_{1}+\cdots+\alpha_{k} \operatorname{deg} Q_{k}=1$ and $\varphi=\left|Q_{1}\right|^{\alpha_{1}} \ldots$ $\left|Q_{k}\right|^{\alpha_{k}}$

(iii) $G_{h}=\operatorname{int} \bigcap_{\varphi \in S\left(\mathbf{C}^{n}\right): \varphi \leqslant h} G_{\varphi}$.

The following two results (Theorems 2.24 and 2.28) are useful to construct new domains of type $O^{(>0)}$.

TheOREM 2.24. Assume that $D \in O^{\left(0^{*}\right)}$. Let $\Omega$ be an $\mathrm{m}$-circled domain in $\mathbf{C}^{m}$ such that $\log \Omega$ is convex. Let $F_{1}, \ldots, F_{m-1}$ be nonzero rational functions of $n$-complex variables, holomorphic in $D$, and let $F_{m} \in O(D), F_{m} \not \equiv 0$. Set $F:=\left(F_{1}, \ldots, F_{m}\right)$ : $D \rightarrow \mathbf{C}^{m}, G:=F^{-1}(\Omega)$. Then $G \in O^{\left(0^{*}\right)}$. In particular, $G \in O^{(>0)}$ iff $G=G^{*}$.

Proof. Fix $N>0$. Suppose that $G^{*}$ is not an $O^{(N)}\left(G^{*}, \delta_{G}\right)$-domain of holomorphy and let $G_{0}, \tilde{G}$ be as in the proof of Theorem 2.18. Let

$$
M:=\left(D^{*} \backslash D\right) \cup\left\{z \in D: F_{1}(z) \cdots F_{m}(z)=0\right\} .
$$

The set $M$ is thin, so there exists $a \in \tilde{G} \backslash(G \cup M)$. The point $F(a)$ belongs to $\mathbf{C}^{m} \backslash \Omega$, so in view of the convexity of $\log \Omega$, there exist $\beta=\left(\beta_{1}, \ldots, \beta_{m}\right) \in\left(\mathbf{R}^{m}\right)_{*}$ and $c>0$ such that

$$
\Omega \subset \Omega_{1}:=\left\{\left|w_{1}\right|^{\beta_{1}} \cdots\left|w_{m}\right|^{\beta_{m}}<c\right\}, \quad F(a) \in \partial \Omega_{1} .
$$

Let $J:=\left\{j \in\{1, \ldots, m\}: \beta_{j}<0\right\}$. Put

$$
\begin{aligned}
& V:=\left\{z \in D: \prod_{j \in J} F_{j}(z)=0\right\}, \quad D_{1}:=D \backslash V ; \\
& u:=\beta_{1} \cdot \ln \left|F_{1}\right|+\cdots+\beta_{m} \cdot \ln \left|F_{m}\right|-\ln c .
\end{aligned}
$$

Note that $\left(D_{1}\right)^{*}=D^{*}, u \in \mathscr{E}\left(D_{1}\right), G_{1}:=\left\{z \in D_{1}: u(z)<0\right\}=F^{-1}\left(\Omega_{1}\right)$.

In view of Corollary $2.19, G_{1} \in O^{\left(0^{*}\right)}$ and hence $\tilde{G} \subset\left(G_{1}\right)^{*}$, so $a \in\left(G_{1}\right)^{*} \cap D_{1}$ $=G_{1}$, which is a contradiction. 
Corollary 2.25. Let $G$ be an n-circled domain such that $\log G$ is convex. Then $G \in O^{\left(0^{*}\right)}$. In particular, $G \in O^{(>0)}$ if and only if $G$ is fat (Corollary 2.7(c)).

Corollary 2.25 generalizes Theorem 1 from [7].

Corollary 2.26. Let $D, \Omega, F, G$ be as in Theorem 2.24. Assume additionally that $D \in O^{(>0)}$ and that $\Omega$ is balanced. Then $G \in O^{(>0)}$.

EXAmple 2.27. Let $D, F$ be as in Theorem 2.24. Put

$$
G:=\left\{z \in D: \sum_{j=1}^{p}\left|F_{1}\right|^{\beta_{j 1}} \cdots\left|F_{m}\right|^{\beta_{j m}}<1\right\},
$$

where $\beta_{j k} \geqslant 0, j=1, \ldots, p, k=1, \ldots, m$. Then $D \in O^{(>0)} \Rightarrow G \in O^{(>0)}$.

For a polynomial automorphism $\Phi: \mathbf{C}^{n} \rightarrow \mathbf{C}^{n}$ the following inequality is well known:

$$
\|z\| \leqslant \alpha\left(1+\|\Phi(z)\|^{\beta}\right) \quad(\alpha, \beta>0) .
$$

Using this information one has for a domain $G \subset \mathbf{C}^{n}$ :

$$
\left(\delta_{\Phi(G)} \cdot \Phi\right)^{k} \leqslant c \cdot \delta_{G} \quad(c, k \text { constants, independent of } G) .
$$

From that the following theorem follows.

THEOREM 2.28. Let $F: \mathbf{C}^{n} \rightarrow \mathbf{C}^{n}$ be a polynomial automorphism. Then, for every $G \in \operatorname{top} \mathbf{C}^{n}$ :

(a) $G \in O^{(0+)}$ iff $F(G) \in O^{(0+)}$.

(b) $G \in O^{(>0)}$ iff $F(G) \in O^{(>0)}$.

3. $n$-circled domains of of type $H^{\infty}, O^{(0+)}, L_{h}^{2}$. Let $G \subset \mathbf{C}^{n}$ be a connected fat and $n$-circled domain of holomorphy. We shall study relations between geometrical properties of the domain $\log G$ and structures of the spaces $H^{\infty}(G), O^{(0+)}(G)$ and $L_{h}^{2}(G)$.

Definition 3.1 (CF. [7]). For a convex domain $X \subset \mathbf{R}^{n}$, let $E(X)$ denote a vector subspace of $\mathbf{R}^{n}$ such that

(a) $X+E(X)=X$,

(b) if $F$ is a vector subspace of $\mathbf{R}^{n}$ such that $X+F=X$ then $\operatorname{dim} F \leqslant \operatorname{dim} E(X)$.

One can easily prove that the space $E(X)$ is uniquely determined and, if $F$ is as in (b), then $F \subset E(X)$. Let $\langle$,$\rangle denote the standard scalar product in \mathbf{R}^{n}$, i.e. $\langle x, y\rangle=x_{1} y_{1}+\cdots+x_{n} y_{n}, x=\left(x_{1}, \ldots, x_{n}\right), y=\left(y_{1}, \ldots, y_{n}\right) \in \mathbf{R}^{n}$.

It is clear that, for every convex domain $X \subsetneq \mathbf{R}^{n}$, there exist $A \subset\left(\mathbf{R}^{n}\right)_{*}$ and a family of points $\left\{x^{\alpha}\right\}_{\alpha \in A}$ such that

$$
X=\operatorname{int} \bigcap_{\alpha \in A} H_{\alpha},
$$

where $H_{\alpha}:=\left\{x \in \mathbf{R}^{n}:\left\langle x-x^{\alpha}, \alpha\right\rangle<0\right\}$. If $X$ is as in (3.1), then one gets

$$
E(X)=\left\{v \in \mathbf{R}^{n}: \forall \alpha \in A:\langle v, \alpha\rangle=0\right\} .
$$


In particular, if

$$
G=\operatorname{int} \bigcap_{\alpha \in A} G_{\alpha}
$$

where $G_{\alpha}:=\left\{z=\left(z_{1}, \ldots, z_{n}\right) \in \mathbf{C}^{n}:\left|z_{1}\right|^{\alpha_{1}} \cdots\left|z_{n}\right|^{\alpha_{n}}<c_{\alpha}\right\}$, then

$$
E(\log G)=\{v: \forall \alpha \in A:\langle v, \alpha\rangle=0\} \quad \text { (cf. Example } 1 \text { in [7]). }
$$

It is well known that every fat $n$-circled domain of holomorphy $G \subset \mathbf{C}^{n}$ can be written in the form (3.2).

Definition 3.2 (CF. [7, Definition 2]). A vector subspace $E \subset \mathbf{R}^{n}$ is said to be of rational type if $E$ is generated by $E \cap \mathbf{Q}^{n}$.

Notice that in concrete situations the condition " $E$ is of rational type" can be effectively verified-cf. [7, Example 2].

Lemmas 4, 5 from [7] imply

LEMMA 3.3. The following conditions are equivalent:

(i) $E$ is of rational type;

(ii) $E^{\perp}$ is of rational type, where $E^{\perp}$ denotes the orthogonal complement of $E$ w.r.t. the scalar product $\langle\cdot, \cdot\rangle$;

(iii) $\operatorname{dim}\left[E^{\perp} \cap \mathbf{Q}^{n}\right]^{\perp}=\operatorname{dim} E$, where $[A]$ denotes the vector subspace of $\mathbf{R}^{n}$ generated by $A$.

By the methods as in the proof of Theorem 2 in [7] we can receive the following result.

Proposition 3.4. Let $X \subsetneq \mathbf{R}^{n}$ be a convex domain, then the following conditions are equivalent:

(i) $E(X)$ is of rational type;

(ii) $X=$ int $\bigcap_{\alpha \in A} H_{\alpha}$, where $A \subset\left(\mathbf{Z}^{n}\right)_{*}(c f$. (3.1));

(iii) For every $x^{0} \notin \bar{X}$ there exists a basis $\alpha^{1}, \ldots, \alpha^{r}$ of $E(X)^{\perp}-r:=\operatorname{codim} E(X)$ - consisting of vectors of $\mathbf{Z}^{n}$ such that $X \subset \cap_{j=1}^{r}\left\{x:\left\langle x-x^{0}, \alpha^{j}\right\rangle<0\right\}$.

Definition 3.5. Let $X$ be a convex domain in $\mathbf{R}^{n}$. Put $D(X):=\left\{z=\left(z_{1}, \ldots, z_{n}\right)\right.$ $\left.\in\left(\mathbf{C}_{*}\right)^{n}: \log z:=\left(\ln \left|z_{1}\right|, \ldots, \ln \left|z_{n}\right|\right) \in X\right\}, \exp X:=\operatorname{int}_{\mathbf{C}^{n}} \operatorname{cl}_{\mathbf{C}^{n}} D(X)$.

In view of Remark 2.6 , one can prove the following lemma.

LemmA 3.6. (a) $X=\log D(X)=\log \exp X$.

(b) $\exp X=D(X)^{*}$.

(c) $\exp X$ is a fat $n$-circled domain of holomorphy.

(d) If $G \subset \mathbf{C}^{n}$ is a fat $n$-circled domain of holomorphy then $G=\exp \log G$.

Definition 3.7. For a convex domain $X \subset \mathbf{R}^{n}$ let

$$
K_{1}(X):=X+\left[E(X)^{\perp} \cap \mathbf{Q}^{n}\right]^{\perp}, \quad K_{j+1}(X):=K_{1}\left(K_{j}(X)\right), \quad j \geqslant 1 .
$$

Put

$$
K(X):=\bigcup_{j=1}^{\infty} K_{j}(X), \quad M(X):=E(K(X))
$$


REMARK 3.8. In view of Lemma 3.3 we have

(a) $E(X)$ is of rational type iff $K_{1}(X)=X$.

(b) If $X \subsetneq \mathbf{R}^{n}$ then there exists $q \leqslant \operatorname{codim} E(X)$ such that $K(X)=K_{q}(X)$.

(c) $M(X)$ is of rational type and $K(X)=X+M(X)$.

(d) $K(X)=\operatorname{int} \cap Y$, where the intersection is taken over all convex domains $Y \subset \mathbf{R}^{n}$ such that $X \subset Y$ and $E(Y)$ is of rational type.

The spaces $H^{\infty}(G)$ and $O^{(0+)}(G)$ are characterized by the following result.

THEOREM 3.9. Let $G \subset \mathbf{C}^{n}$ be an n-circled domain of holomorphy. Then the following conditions are equivalent:

(i) $G \in H^{\infty}$;

(ii) $G \in O^{(0+)}$;

(iii) $G$ is fat and the space $E(\log G)$ is of rational type;

(iv) $G=\operatorname{int} \bigcap_{\alpha \in A} G_{\alpha}$; where $A \subset \mathbf{Z}^{n}$ (cf. (3.2)).

Theorem 3.9 implies Theorem 2 in [7], and Theorem 7.1 and Corollary in $§ 7$ of [14].

Proof. In the case when $G$ is balanced, the proof of (i) $\Leftrightarrow$ (iii) was given in [7, Theorem 2]. The same method of the proof can be applied to the general case -cf. [14, Corollary in \$7]. In view of Proposition 3.4, one has: (iii) $\Leftrightarrow$ (iv). It remains to show that (ii) $\Rightarrow$ (iii). By Corollary 2.7 (c), $G$ is fat. Put $X:=\log G, F:=$ $\left[E(X)^{\perp} \cap \mathbf{Q}^{n}\right]^{\perp}$ and suppose that $E(X)$ is not of rational type. Then, according to Lemma 3.3 , it is clear that

$$
\operatorname{dim} F>\operatorname{dim} E(X) .
$$

Let $f \in O^{(0+)}(G)$ be a nonextendable function and let

$$
\sum_{\nu \in \mathbf{Z}^{n}} a_{\nu} z^{\nu}
$$

be the $n$-fold Laurent series expansion of $f$. We shall show that

$$
a_{\nu}=0, \quad \nu \notin E(X)^{\perp} .
$$

Suppose for a moment that (3.5) holds true. Then for $x \in X, v \in F$ we get

$$
\sum_{\nu \in \mathbf{Z}^{n}} a_{\nu}\left(e^{x+v}\right)^{\nu}=\sum_{\nu \in E(X)^{\perp} \cap \mathbf{Z}^{n}} a_{\nu}\left(e^{x}\right)^{\nu} .
$$

Consequently, the series (3.4) is convergent in the domain $D\left(K_{1}(X)\right)$. Since the function $f$ is not continuable, $K_{1}(X) \subset X$. Thus $X+F \subset X$ which contradicts (3.3).

We pass to the proof of (3.5). Fix $\nu \notin E(X)^{\perp}$ and let $w \in E(X)$ be such that $\langle w, \nu\rangle>0,\|w\|=1$. Fix $0<N<\langle w, \nu\rangle$ and $x^{0} \in X$. Set $x(t):=e^{x^{0}+t w}, t \in \mathbf{R}$. Since $x^{0}+t w \in X, t \in \mathbf{R}$, the Cauchy inequalities imply

$$
\begin{aligned}
\left|a_{\nu}\right| & \leqslant\left[\sup \left\{\left|f\left(z_{1}, \ldots, z_{n}\right)\right|:\left|z_{j}\right|=x_{j}(t), j=1, \ldots, n\right\}\right] \cdot[x(t)]^{-\nu} \\
& \leqslant\left\|\delta_{G}^{N} f\right\|_{\infty} \cdot\left\{\delta_{G}^{N}(x(t)) \cdot[x(t)]^{\nu}\right\}^{-1} \\
& \leqslant c\left[\delta_{G}(x(t)) \cdot \exp (t / N \cdot\langle w, \nu\rangle)\right]^{-N}, \quad t \in \mathbf{R},
\end{aligned}
$$


where $c>0$ is a constant independent of $t$. Put

$$
M:=\sup \left\{\delta_{G}(x(t)) \cdot \exp ((t / N)\langle w, \nu\rangle): t \in \mathbf{R}\right\} .
$$

In order to show that $a_{\nu}=0$ it is enough to prove that $M=+\infty$. Suppose that $M<+\infty$ and let

$$
T:=\left\{t>0: \rho_{G}(x(t))>\delta_{0}(x(t))\right\} .
$$

If $t \in T$ then

$$
\exp \left(\frac{-2 t}{N}\langle w, \nu\rangle\right)+\sum_{j=1}^{n} \exp \left[2\left(x_{j}^{0}+t w_{j}-\frac{t}{N}\langle w, v\rangle\right)\right] \geqslant \frac{1}{M^{2}}, \quad t \in T .
$$

Consequently the set $T$ is bounded, that is there exists a $t_{0}>0$ such that

$$
\delta_{G}(x(t))=\rho_{G}(x(t)), \quad t \geqslant t_{0} .
$$

Let

$$
d:=\operatorname{dist}\left(x^{0}+\mathbf{R} w, \partial X\right)=\operatorname{dist}\left(x^{0}, \partial X\right) .
$$

Fix $t \in \mathbf{R}$ and let $z=\left(z_{1}, \ldots, z_{n}\right) \in \partial G, z_{1} \cdots z_{n} \neq 0$ be such that

$$
\rho_{G}(x(t)) \geqslant \frac{1}{2}\|z-x(t)\| \text {. }
$$

We can write $\log z=x^{0}+t w+u$ with $u \in \mathbf{R}^{n},\|u\| \geqslant d$. Let $j=j(t)$ be such that $\left|u_{j}\right| \geqslant d / \sqrt{n}$. It is seen that

$$
\rho_{G}(x(t)) \geqslant \frac{1}{2}\left|z_{j}-x_{j}(t)\right| \geqslant \frac{1}{2} \exp \left(x_{j}^{0}+t w_{j}\right)\left|e^{u_{j}}-1\right| \geqslant d_{0} \exp \left(x_{j}^{0}+t w_{j}\right),
$$

where $d_{0}>0$ is a constant independent of $t$. Let $j_{0} \in\{1, \ldots, n\}$ be such that there exists a sequence $\left(t_{k}\right)_{k=1}^{\infty}$ with $t_{k} \geqslant t_{0}, j\left(t_{k}\right)=j_{0}, k \geqslant 1, t_{k} \rightarrow+\infty$. Then

$$
\begin{aligned}
+\infty & >M \geqslant \rho_{G}\left(x\left(t_{k}\right)\right) \cdot \exp \left(\left(t_{k} / N\right)\langle w, \nu\rangle\right) \\
& \geqslant d_{0} \cdot \exp \left(x_{j_{0}}^{0}+t_{k} w_{j_{0}}+\left(t_{k} / N\right)\langle w, \nu\rangle\right) \rightarrow+\infty \quad \text { as } k \rightarrow+\infty .
\end{aligned}
$$

This contradiction proves $M=\infty$.

REMARK. In view of 1.5 one can formulate the following question-cf. [15, §5]. Let $G=G_{h}$ be a bounded balanced domain of holomorphy such that $h$ is continuous (cf. §2). Does this imply that $G$ is Carathéodory finitely compact? (It is known that the continuity of $h$ is necessary.)

REMARK 3.10. In view of the Banach theorem, either $H^{\infty}(G)=O^{(0+)}(G)$ or $H^{\infty}(G)$ is of the first Baire category in $O^{(0+)}(G)$.

Proposition 3.11. Let $G \subsetneq \mathbf{C}^{n}$ be an n-circled domain of type $H^{\infty}$. Then $H^{\infty}(G) \varsubsetneqq$ $O^{(0+)}(G)$ (cf. the implication (ii) $\Rightarrow$ (i) in Theorem 3.9 and Remark 3.10).

Proof. In view of the implication (i) $\Rightarrow$ (iv) in Theorem 3.9 there exists an $\alpha \in\left(\mathbf{Z}^{n}\right)_{*}$ such that $G \subset G_{\alpha}, \partial G \cap \partial G_{\alpha} \cap\left(\mathbf{C}_{*}\right)^{n} \neq \varnothing$. Fix $a=\left(a_{1}, \ldots, a_{n}\right) \in \partial G$ $\cap \partial G_{\alpha}, a_{1} \cdots a_{n} \neq 0$. It suffices to show that there exists an $f \in O^{(0+)}\left(G_{\alpha}\right)$ such that $\lim _{z \rightarrow a} f(z)=\infty$. The result is a direct consequence of the following lemma.

LemMA 3.12. Let $L, M$ be two nonzero complex polynomials of $n$-complex variables with $\operatorname{deg} L+\operatorname{deg} M \geqslant 1$. Set

$$
\Omega:=\left\{z \in \mathbf{C}^{n}:|L(z)|<|M(z)|\right\} .
$$


Then for every $a \in(\partial \Omega) \backslash M^{-1}(0)$ there exists $f \in O^{(0+)}(\Omega)$ such that $\lim _{z \rightarrow a} f(z)=$ $\infty$. In particular $H^{\infty}(\Omega) \varsubsetneqq O^{(0+)}(\Omega)($ cf. Remark 3.10).

Notice that $\Omega \in H^{\infty}$ provided $L, M$ have no common factor.

Proof. Let

$$
\varphi(\lambda):=\log 1 /(1-\lambda), \quad \lambda \in \mathbf{D}:=\{\lambda \in \mathbf{C}:|\lambda|<1\} .
$$

Observe that $\lim _{\lambda \rightarrow 1} \varphi(\lambda)=\infty$ and

(3.6) for every $\varepsilon>0$ the function $(1-\lambda)|\varphi|^{1 / \varepsilon}$ is bounded in D. Fix $a \in$ $(\partial \Omega) \backslash M^{-1}(0)$ and let $\lambda_{0}:=M(a) / L(a)$. Define

$$
f(z)=\varphi\left(\lambda_{0} \cdot L(z) / M(z)\right), \quad z \in \Omega .
$$

It is clear that $\lim _{z \rightarrow a} f(z)=\infty$. It remains to show that $f \in O^{(0+)}(G)$. Fix $N>0$. Set $R:=M-\lambda_{0} L, d:=\operatorname{deg} R, d_{0}:=\operatorname{deg} M, \varepsilon:=N / d+d_{0}$. In view of (3.6), there exists a constant $c>0$ such that

$$
\delta_{0}^{\left.d_{0}\right)} \cdot|R| \cdot|f|^{1 / \varepsilon}=\delta_{0}^{d_{0}} \cdot|M| \cdot\left|1-\lambda_{0} \frac{L}{M}\right| \cdot\left|\varphi\left(\lambda_{0} \frac{L}{M}\right)\right|^{1 / \varepsilon} \leqslant c\left\|\delta_{0}^{d_{0}} \cdot M\right\|_{\infty} .
$$

Hence, by Lemma $2.17, f \in O^{(N)}\left(\Omega, \delta_{\Omega}\right)$.

THEOREM 3.13. Let $G$ be an $n$-circled domain such that $X:=\log G$ is convex. Then the domain $\exp K(\log G)$ is the $H^{\infty}$-envelope of holomorphy of $G$. In particular:

(a) $H^{\infty}(G) \simeq \mathbf{C}$ iff $K(\log G)=\mathbf{R}^{n}$.

(b) For every $f \in H^{\infty}(G)$, the Laurent series of $f$ is of the form $\sum_{\nu \in M(X)^{\perp} \cap \mathbf{z}^{n}} a_{\nu} z^{\nu}$ (cf. the proof of the implication (ii) $\Rightarrow$ (iii) in Theorem 3.9).

Proof. Put $\tilde{G}:=\exp K(X)$. In view of Lemma 3.6(a) and Remark 3.8(c), $\tilde{G}$ is a fat $n$-circled domain such that the space $E(\log \tilde{G})=M(X)$ is of rational type. Hence, according to Theorem 3.9, $\tilde{G}$ is the domain of existence of a bounded holomorphic function. It remains to show that $H^{\infty}(G) \subset O(\tilde{G})$. In view of Lemma 3.6(b) and Corollary 2.7(a), it suffices to prove that $H^{\infty}(G) \subset H^{\infty}(D(K(X)))$. As in the proof of the implication (ii) $\Rightarrow$ (iii) in Theorem 3.9, one can prove that for every $f \in H^{\infty}(G)$,

$$
f(z)=\sum_{\nu \in E(X)^{\perp} \cap \mathbf{Z}^{n}} a_{\nu} z^{\nu}
$$

thus $f$ extends holomorphically to a function from $O\left(D\left(K_{1}(X)\right)\right)$. Hence $H^{\infty}(G) \subset$ $H^{\infty}\left(D\left(K_{1}(X)\right)\right)$. Now our arguments can be repeated and they prove that $H^{\infty}(G) \subset$ $\left.H^{\infty}\left(D\left(K_{2}(X)\right)\right)\right)$. After a finite number of steps we get the result.

Let $G \varsubsetneqq \mathbf{C}^{n}$ be an $n$-circled domain of type $H^{\infty}$. Put $X:=\log G, r:=\operatorname{codim} E(X)$ and assume that $r \leqslant n-1$. In view of Theorem 1, Chapter VII from [2], there exists a basis $\alpha^{1}, \ldots, \alpha^{r}$ of $E(X)^{\perp}$ consisting of vectors from $\mathbf{Z}^{n}$ such that

$$
E(X)^{\perp} \cap \mathbf{Z}^{n}=\mathbf{Z} \boldsymbol{\alpha}^{1}+\cdots+\mathbf{Z} \boldsymbol{\alpha}^{r} .
$$

Let $L: \mathbf{R}^{n} \rightarrow \mathbf{R}^{r}$ be a linear mapping given by the formula

$$
L(x)=\left(\left\langle x, \alpha^{1}\right\rangle, \ldots,\left\langle x, \alpha^{r}\right\rangle\right), \quad x \in \mathbf{R}^{n} .
$$

Observe that $\left.L\right|_{E(X)^{\perp}}$ is an isomorphism of $E(X)^{\perp}$ onto $\mathbf{R}^{r}$. 
Let $Y:=L(X)$. It is easily seen that $Y$ is a convex domain such that $E(Y)=\{0\}$. Put $\Omega:=\exp Y ; \Omega$ is an $r$-circled domain of type $H^{\infty}$ (Theorem 3.9). Let $\Phi=$ $\left(\Phi_{1}, \ldots, \Phi_{r}\right):\left(\mathbf{C}_{*}\right)^{n} \rightarrow\left(\mathbf{C}_{*}\right)^{r}$ be given by the formula $\Phi_{j}(z)=z^{\alpha^{j}}, j=1, \ldots, r$. We shall show that

$$
\Phi(D(X))=D(Y) .
$$

Note that $\log \Phi(D(X))=L(X)=Y=\log D(Y)$. Now it suffices to show that the set $\Phi(D(X))$ is $r$-circled. Fix $z \in D(X), \theta \in \mathbf{R}^{r}$. In view of surjectivity of $L$, there exists $\eta \in \mathbf{R}^{n}$ such that $L(\eta)=\theta$. Then

$$
\Phi_{j}\left(e^{i \eta_{1}} z_{1}, \ldots, e^{i \eta_{n}} z_{n}\right)=e^{i\left\langle\eta, \alpha^{j}\right\rangle} z^{\alpha^{j}}=e^{i \theta_{j}} \Phi_{j}(z), \quad j=1, \ldots, r,
$$

and therefore $\left(e^{i \theta_{1}} \Phi_{1}(z), \ldots, e^{i \theta_{r}} \Phi_{r}(z)\right) \in \Phi(D(X))$.

Because of (3.8), the operator $T: H^{\infty}(D(Y)) \rightarrow H^{\infty}(D(X))$ given by the formula $T \varphi=\varphi \circ \Phi, \quad \varphi \in H^{\infty}(D(Y))$ is a well-defined isometry of $H^{\infty}(D(Y))$ into $H^{\infty}(D(X))$. We shall show that, in fact, $T$ is an isomorphism. Fix $f=\sum_{\nu \in \mathbf{Z}} a_{\nu} z^{\nu} \in$ $H^{\infty}(D(X))$. Then, in view of (3.7),

$$
f(z)=\sum_{\nu \in E(X)^{\perp} \cap \mathbf{Z}^{n}} a_{\nu} z^{\nu}=\sum_{\beta \in \mathbf{Z}^{r}} a_{\nu(\beta)}\left(z^{\alpha^{1}}\right)^{\beta_{1}} \cdots\left(z^{\alpha^{r}}\right)^{\beta_{r}},
$$

where $\nu(\beta):=\beta_{1} \alpha^{1}+\cdots+\beta_{r} \alpha^{r}$. Define

$$
\varphi(\lambda)=\sum_{\beta \in \mathbf{Z}^{r}} a_{\nu(\beta)} \lambda^{\beta}, \quad \lambda \in D(Y) .
$$

By dint of (3.8), $\varphi$ is well defined and $\|\varphi\|_{\infty}=\|f\|_{\infty}$. It is clear that $T \varphi=f$.

In view of Corollary 2.7(a) and Lemma 3.6(b), (d), the operator $T$ extends uniquely to an isometrical isomorphism of $H^{\infty}(\Omega)$ onto $H^{\infty}(G)$.

Thus we have proved the following

- THeOReM 3.14. Let $G \varsubsetneqq \mathbf{C}^{n}$ be an n-circled domain of type $H^{\infty}$ such that $r:=\operatorname{codim} E(\log G) \leqslant n-1$. Then there exists an r-circled domain $\Omega$ of type $H^{\infty}$ with $E(\log \Omega)=\{0\}$ such that the spaces $H^{\infty}(\Omega)$ and $H^{\infty}(G)$ are isometrically isomorphic.

Remark 3.15. Let $G$ be as in Theorem 3.14 and let $c_{G}$ denote the Carathéodory pseudodistance on $G$. Define for $x, y \in G: x \sim y$ iff $c_{G}(x, y)=0$. Then Theorem 3.14 gives a complex structure of $\left[G \cap\left(\mathbf{C}_{*}\right)^{n}\right] / \sim$.

EXAMPLE 3.16. Let $G:=\left\{z \in \mathbf{C}^{3}:\left|z_{1}\right|^{2} \cdot\left|z_{3}\right|<1,\left|z_{2}\right|^{2} \cdot\left|z_{3}\right|<1\right\}$. Following the proof of Theorem 3.14 one gets

$$
\begin{aligned}
& E(X)^{\perp}=\mathbf{R}(2,0,1)+\mathbf{R}(0,2,1)=: \mathbf{R} \nu^{1}+\mathbf{R} \nu^{2}, \\
& E(X)^{\perp} \cap \mathbf{Z}^{3}=\mathbf{Z} \cdot(1,1,1)+\mathbf{Z} \cdot(1,-1,0)=: \mathbf{Z} \alpha^{1}+\mathbf{Z} \alpha^{2}
\end{aligned}
$$

and therefore one has

$$
\Omega=\left\{\lambda \in \mathbf{C}^{2}:\left|\lambda_{1} \lambda_{1}\right|<1,\left|\lambda_{1} / \lambda_{2}\right|<1\right\} \quad \text { and } \quad \Phi(z)=\left(z_{1} \cdot z_{2} \cdot z_{3}, z_{1} / z_{2}\right) .
$$


Observe that $G$ is balanced but $\Omega$ is not. According to this example one can pose the following question: Are there a balanced 2-circled domain of holomorphy $\tilde{\Omega} \subset \mathbf{C}^{2}$ and $\tilde{\alpha}^{1}, \tilde{\alpha}^{2} \in\left(\mathbf{Z}_{+}\right)_{*}^{3}$, such that

$$
\begin{gathered}
E(\log \tilde{\Omega})=\{0\}, \\
\tilde{\Phi}(G) \subset \tilde{\Omega} \quad\left(\tilde{\Phi}(z)=\left(z^{\tilde{\alpha}^{1}}, z^{\tilde{\alpha}^{2}}\right)\right)
\end{gathered}
$$

and the mapping

$$
H^{\infty}(\tilde{\Omega}) \ni f \rightarrow f \circ \tilde{\Phi} \in H^{\infty}(G) \text { is surjective. }
$$

In order to give the negative answer let us assume that $\tilde{\Omega}, \tilde{\alpha}^{1}, \tilde{\alpha}^{2}$ are as above.

By (3.9) and (3.10) one gets $\tilde{\alpha}^{1}, \tilde{\alpha}^{2} \in[E(X)]^{\perp}$. Since $z_{1}^{2} z_{3}, z_{2}^{2} z_{3}, z_{1} z_{2} z_{3}$ belong to $H^{\infty}(G)$, by (3.11), we have $\alpha^{1}, \nu^{1}, \nu^{2} \in \mathbf{Z}_{+} \tilde{\alpha}_{1}+\mathbf{Z}_{+} \cdot \tilde{\alpha}_{1}$. Then calculation gives $\alpha^{1} \in \mathbf{Z}_{+} \cdot \nu^{1}+\mathbf{Z}_{+} \cdot \nu^{2}$ which is impossible. We would like to thank S. Cynk (Jagellonian University) for suggesting this kind of example.

We shall show how the domain $K(\log G)$ can be effectively calculated in the case when $G$ is a finite intersection of elementary $n$-circled domains.

Let us start with a lemma we will need in the proof of Proposition 3.18.

Lemma 3.17. Let $X_{0}, \ldots, X_{N}$ be open half-spaces in $\mathbf{R}^{n}$ and let $\boldsymbol{\alpha}^{j} \perp \partial X_{j}, \boldsymbol{\alpha}^{j} \in\left(\mathbf{R}^{n}\right)_{*}$ $(j=0, \ldots, N)$ with $r:=\operatorname{rank}\left(\alpha^{1}, \ldots, \alpha^{N}\right)$ and $\varnothing \neq X_{1} \cap \cdots \cap X_{N} \subset X_{0}$. Then there exists $1 \leqslant i_{1}<\cdots<i_{r} \leqslant N$ such that

$$
r=\operatorname{rank}\left(\alpha^{i_{1}}, \ldots, \alpha^{i_{r}}\right) \text { and } X_{i_{1}} \cap \cdots \cap X_{i_{r}} \subset X_{0} .
$$

For convenience a proof will be given in the Appendix.

Let $G=G_{1} \cap \cdots \cap G_{N}$, where $G_{j}=\left\{z:\left|z_{1}\right|_{1}^{\alpha_{1}^{j}} \cdots\left|z_{n}\right|^{\alpha_{n}^{j}}<c_{j}\right\}, \alpha^{j}=\left(\alpha_{1}^{j}, \ldots, \alpha_{n}^{j}\right)$ $\in\left(\mathbf{R}^{n}\right)_{*}, c_{j}>0, j=1, \ldots, N$.

Put $X:=\log G, r:=\operatorname{rank}\left[\alpha^{1}, \ldots, \alpha^{N}\right]$,

$$
\begin{aligned}
& J:=\left\{I=\left(i_{1}, \ldots, i_{r}\right): 1 \leqslant i_{1}<\cdots<i_{r} \leqslant N, \operatorname{rank}\left[\alpha^{i_{1}}, \ldots, \alpha^{i_{r}}\right]=r\right\}, \\
& A_{I}:=\mathbf{Z}^{n} \cap\left(\mathbf{R}_{+} \alpha^{i_{1}}+\cdots+\mathbf{R}_{+} \alpha^{i_{r}}\right), \\
& M_{I}:=\left\{v: \forall \alpha \in A_{I}:\langle v, \alpha\rangle=0\right\}, \quad I=\left(i_{1}, \ldots, i_{r}\right) \in J, \\
& M:=\bigcap_{I \in J} M_{I} .
\end{aligned}
$$

Proposition 3.18. With the notation above, we have $M(X)=M$ and, consequently, $K(X)=X+M$. In particular

$$
H^{\infty}(G) \simeq \mathrm{C} \text { iff for every } I \in J: A_{I}=\{0\} \quad(c f . \text { Theorem 3.13(a)). }
$$

Proof. Let $X_{j}:=\log G_{j}, j=1, \ldots, N$,

$$
X_{I}:=\bigcap_{k=1}^{r} X_{i_{k}}, \quad I=\left(i_{1}, \ldots, i_{r}\right) \in J
$$

For every $I=\left(i_{1}, \ldots, i_{r}\right) \in J$, there exists a point $x^{I} \in \mathbf{R}^{n}$ such that $X_{i_{k}}=\{x$ : $\left.\left\langle x-x^{l}, \alpha^{i_{\Lambda}}\right\rangle<0\right\}, k=1, \ldots, r$.

Define

$$
Y_{I}=\operatorname{int} \bigcap_{\alpha \in A_{I}}\left\{x:\left\langle x-x^{I}, \alpha\right\rangle<0\right\}, \quad I \in J .
$$


Observe that $X \subset X_{I} \subset Y_{I}$ and $E\left(Y_{I}\right)=M_{I}$. By Proposition 3.4, $E\left(Y_{I}\right)$ is of rational type, so $M(X) \subset M\left(Y_{I}\right)=M_{I}, I \in J$, and therefore $M(X) \subset M$.

If $M(X)=\mathbf{R}^{n}$, then the proof is finished, so assume that $K(X)=\operatorname{int} \bigcap_{\beta \in B} H_{\beta}$, where $B \subset\left(\mathbf{Z}^{n}\right)_{*}$, and hence $M(X)=\{v: \forall \beta \in B:\langle v, \beta\rangle=0\}$. We shall show that

$$
B \subset \bigcup_{I \in J} A_{I} .
$$

Note that (3.12) implies immediately that $M \subset M(X)$.

Fix $\beta \in B$. By Lemma 3.17 there exists an $I=\left(i_{1}, \ldots, i_{r}\right) \in J$ such that

$$
X_{I} \subset H_{\beta}=\left\{x:\left\langle x-x^{\beta}, \beta\right\rangle<0\right\} .
$$

Hence $\beta \in E\left(H_{\beta}\right)^{\perp} \subset E\left(X_{I}\right)^{\perp}=\mathbf{R} \alpha^{i_{1}}+\cdots+\mathbf{R} \alpha^{i_{r}}$ and therefore $\beta=\lambda_{1} \alpha^{i_{1}}$ $+\cdots+\lambda_{r} \alpha^{i_{r}}$, where $\lambda:=\left(\lambda_{1}, \ldots, \lambda_{r}\right) \in\left(\mathbf{R}^{r}\right)_{*}$. It remains to show that $\lambda \in\left(\mathbf{R}_{+}\right)^{r}$.

Let $L: \mathbf{R}^{n} \rightarrow \mathbf{R}^{r}$ be given by the formula

$$
L(x)=\left(\left\langle x, \alpha^{i_{1}}\right\rangle, \ldots,\left\langle x, \alpha^{i_{r}}\right\rangle\right), \quad x \in \mathbf{R}^{n} .
$$

Observe that

$$
L\left(X_{I}\right)=\left\{\xi=\left(\xi_{1}, \ldots, \xi_{r}\right) \in \mathbf{R}^{r}: \xi_{k}<\left\langle x^{I}, \alpha^{i_{k}}\right\rangle, k=1, \ldots, r\right\}
$$

and

$$
L\left(H_{\beta}\right)=\left\{\xi:\left\langle\xi-L\left(x^{\beta}\right), \lambda\right\rangle<0\right\} .
$$

The inclusion $L\left(X_{I}\right) \subset L\left(H_{\beta}\right)$ implies that $\lambda \in\left(\mathbf{R}_{+}\right)^{r}$ which gives the required result.

EXAMPLE 3.19. Let

$$
G:=\left\{\left(z_{1}, z_{2}, z_{3}\right) \in \mathbf{C}^{3}:\left|z_{1}\right|^{\alpha} \cdot\left|z_{2}\right|^{\alpha+1}<1,\left|z_{1}\right|^{\alpha+1} \cdot\left|z_{2}\right|^{\alpha+2} \cdot\left|z_{3}\right|<1\right\},
$$

where $\alpha \in \mathbf{R}_{>0} \backslash \mathbf{Q}$. Put $\alpha^{1}:=(\alpha, \alpha+1,0), \alpha^{2}:=(\alpha+1, \alpha+2,1)$. Clearly $\operatorname{rank}\left[\alpha^{1}, \alpha^{2}\right]=2$. One can easily prove that

$$
\mathbf{Z}^{3} \cap\left(\mathbf{R}_{+} \alpha^{1}+\mathbf{R}_{+} \alpha^{2}\right)=\{0\} .
$$

Hence, by Proposition 3.18, $K(\log G)=\mathbf{R}^{3}$ and therefore $H^{\infty}(G) \simeq \mathbf{C}$.

ExAmple 3.20. Let

$$
G:=\left\{\left(z_{1}, z_{2}, z_{3}\right) \in \mathbf{C}^{3}:\left|z_{1}\right|^{\alpha_{1}} \cdot\left|z_{2}\right|^{\alpha_{2}} \cdot\left|z_{3}\right|^{\alpha_{3}}<1,\left|z_{1}\right|^{1-\alpha_{1}} \cdot\left|z_{2}\right|^{1-\alpha_{2}} \cdot\left|z_{3}\right|^{1-\alpha_{3}}<1\right\},
$$

where $0<\alpha_{1}, \alpha_{2}, \alpha_{3}<1, \alpha_{1} \neq \alpha_{2},\left(\alpha_{1}-\alpha_{3}\right) /\left(\alpha_{1}-\alpha_{2}\right) \notin \mathbf{Q}$. Put $\alpha^{1}:=\left(\alpha_{1}, \alpha_{2}, \alpha_{3}\right)$, $\alpha^{2}:=\left(1-\alpha_{1}, 1-\alpha_{2}, 1-\alpha_{3}\right)$. Note that $\operatorname{rank}\left[\alpha^{1}, \alpha^{2}\right]=2$. It can be easily verified that

$$
\mathbf{Z}^{3} \cap\left(\mathbf{R}_{+} \boldsymbol{\alpha}^{1}+\mathbf{R}_{+} \boldsymbol{\alpha}^{2}\right)=\mathbf{Z}_{+} \cdot(1,1,1) .
$$

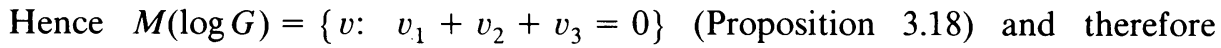
$K(\log G)=\left\{x: x_{1}+x_{2}+x_{3}<0\right\}$. By Theorems 3.13 and 3.14 we have $H^{\infty}(G) \simeq$ $H^{\infty}(\mathbf{D})$.

Now we pass to the characterization of the space of square integrable holomorphic functions. 
THEOREM 3.21. Let $G \varsubsetneqq \mathbf{C}^{n}$ be a fat n-circled domain of holomorphy. Then the following conditions are equivalent:

(i) $L_{h}^{2}(G) \neq\{0\}$;

(ii) $E(\log G)=\{0\}$;

(iii) $G \in L_{h}^{2}$;

(iv) for every $a=\left(a_{1}, \ldots, a_{n}\right) \notin \bar{G}$ with $a_{1} \cdots a_{n} \neq 0$, there exist sequences $\left(c_{\mu}\right)_{\mu=1}^{\infty} \subset \mathbf{R}_{>0},(\nu(\mu))_{\mu=1}^{\infty} \subset \mathbf{Z}^{n}\left(\nu(\mu) \in\left(\mathbf{Z}_{+}\right)^{n}, \mu \in \mathbf{N}\right.$, provided that $G$ is balanced) such that

$$
\left\|c_{\mu} z^{\nu(\mu)}\right\|_{L^{2}(G)} \leqslant 1, \quad \mu \geqslant 1, \quad \lim _{\mu \rightarrow+\infty} c_{\mu}\left|a^{\nu(\mu)}\right|=+\infty .
$$

Proof. The proof of (i) $\Rightarrow$ (ii) is as in [7, Theorem 3]. It is clear that (iv) $\Rightarrow$ (iii) $\Rightarrow$ (i). It remains to prove that (ii) $\Rightarrow$ (iv). Fix $a, \mu$ and let $X:=\log G, x^{0}:=\log a$. Note that $x^{0} \notin \bar{X}$. Let $\alpha^{1}, \ldots, \alpha^{n}$ be as in Proposition 3.4(iii) $(r=n)$. Note that $\alpha^{1}, \ldots, \alpha^{n}$ $\in\left(\mathbf{Z}_{+}\right)^{n}$ provided $G$ is balanced. Let $A:=\left[\alpha_{k}^{j}\right]_{j, k=1, \ldots, n}$. Without loss of generality we assume that $|\operatorname{det} A| \geqslant \mu^{2} \pi^{n}\left|a_{1} \cdots a_{n}\right|^{2}$. Set $\nu(\mu):=\alpha^{1}+\cdots+\alpha^{n}-\mathbf{1}$, where $1:=(1, \ldots, 1) \in \mathbf{N}^{n}$. Now, one can prove (cf. the proof of Theorem 3 in [7]) that

$$
\left\|z^{\nu(\mu)}\right\|_{L^{2}(G)} \leqslant \pi^{n / 2}|\operatorname{det} A|^{-1 / 2}\left|a^{\alpha^{1}+\cdots+\alpha^{n}}\right| \leqslant \frac{1}{\mu}\left|a^{\nu(\mu)}\right| .
$$

Let $c_{\mu}:=\mu\left|a^{-\nu(\mu)}\right|$. Then

$$
\left\|c_{\mu} z^{\nu(\mu)}\right\|_{L^{2}(G)} \leqslant 1 \quad \text { and } \quad c_{\mu}\left|a^{\nu(\mu)}\right|=\mu .
$$

REMARK. One may ask for a characterization of those balanced domains of holomorphy $G$ for which $L_{h}^{2}(G) \neq\{0\}$ or, even more, such that $G \in L_{h}^{2}-$ cf. $\S 5$ in [15].

\section{Appendix.}

Proof of Lemma 3.17. Because the result is clear for $n=1$, let us assume that it is true in $\mathbf{R}^{1}, \ldots, \mathbf{R}^{n-1}$. Without loss of generality we assume that $N \geqslant 2$ and, for $k=1, \ldots, N, \cap_{j \neq k} X_{k} \not \subset X_{0}$. Now, it suffices to prove that $r=N$.

Step 1: $r<n$.

By an orthogonal transformation of the coordinates we can assume that

$$
\boldsymbol{\alpha}^{j}=\left(\tilde{\alpha}^{j}, 0\right) \in \mathbf{R}^{r} \times\{0\}, \quad 0 \leqslant j \leqslant N .
$$

The set $X_{j} \cap\left(\mathbf{R}^{r} \times\{0\}\right)$ can be identified with an open halfspace $\tilde{X}_{j}$ in $\mathbf{R}^{r}$, $j=0, \ldots, N$. It is clear that

$$
r=\operatorname{rank}\left(\tilde{\boldsymbol{\alpha}}^{1}, \ldots, \tilde{\boldsymbol{\alpha}}^{N}\right), \quad \varnothing \neq \bigcap_{j=1}^{N} \tilde{X}_{j} \subset \tilde{X}_{0}, \bigcap_{\substack{j=1 \\ j \neq k}}^{N} \tilde{X}_{j} \not \subset \tilde{X}_{0} .
$$

Therefore one gets $r=N$.

Step 2: $r=n$.

Without loss of generality we assume $X_{0}:=\left\{x \in \mathbf{R}^{n}: x_{n}<0\right\}$. Let $\alpha^{j}=\left(\tilde{\alpha}^{j}, \alpha_{n}^{j}\right)$ $\in \mathbf{R}^{n-1} \times \mathbf{R}, j=1, \ldots, N$. By our assumption we have $\tilde{\boldsymbol{\alpha}}^{j} \neq 0$ for $j=1, \ldots, N$.

Let $\tilde{X}_{0}:=\partial X_{0} \cap\left(\mathbf{R}^{n} \backslash \bar{X}_{N}\right)$ and $\tilde{X}_{j}:=\partial X_{0} \cap X_{j}, j=1, \ldots, N-1$. Then $\tilde{X}_{j}$, $j=0, \ldots, N-1$, can be regarded as open halfspaces in $\mathbf{R}^{n-1}$ with $\tilde{\alpha}^{N} \perp \tilde{X}_{0}$, $\tilde{\boldsymbol{\alpha}}^{j} \perp \tilde{X}_{j}$ for $j=1, \ldots, N-1$ and $\varnothing \neq \bigcap_{j=1}^{N-1} \tilde{X}_{j} \subset \tilde{X}_{0}$. 
Let $\tilde{r}:=\operatorname{rank}\left(\tilde{\alpha}^{1}, \ldots, \tilde{\alpha}^{N-1}\right)$; then, by induction, there exist $1 \leqslant i_{1}<\cdots<i_{\tilde{r}} \leqslant$ $N-1$ such that $\tilde{X}_{i_{1}} \cap \cdots \cap \tilde{X}_{i_{\tilde{r}}} \subset \tilde{X}_{0}$; hence $X_{i_{1}} \cap \cdots \cap X_{i_{\tilde{r}}} \cap X_{N} \subset X_{0}$ and so $N=\tilde{r}+1$. Since $\tilde{r} \leqslant n-1=r-1$ we have $r=N$. This completes the proof.

We like to mention that the idea of Step 2 is due to P. Jakóbczak.

Proof of Lemma 2.17. It is known that there exists a unitary isomorphism $T$ : $\mathbf{C}^{n} \rightarrow \mathbf{C}^{n}$ such that the projection $T\left(R^{-1}(0)\right) \ni\left(w^{\prime}, w_{n}\right) \rightarrow w^{\prime} \in \mathbf{C}^{n-1}$ is proper (cf. [16]).

Put $\hat{G}=T(G), \hat{\varphi}=\varphi \circ\left(\left.T^{-1}\right|_{\hat{G}}\right), \hat{R}=R \circ T^{-1}$. Observe that $\delta_{\hat{G}} \circ T=\delta_{G}$, then

$$
\delta_{\hat{G}}^{\mu}|R| \hat{\varphi} \leqslant\left\|\delta_{G}^{\mu} \cdot R \cdot \varphi\right\|_{\infty}=: c \quad \text { on } \hat{G} .
$$

In view of [16, Lemma 1] the polynomial $\hat{R}$ has the form

$$
\hat{R}\left(w^{\prime}, w_{n}\right)=\sum_{i=0}^{\nu} \hat{R}_{j}\left(w^{\prime}\right) w_{n}^{\nu-j}
$$

where $\nu \leqslant d$ and $\hat{R}_{0}=$ const $\neq 0$.

Fix $w=\left(w^{\prime}, w_{n}\right) \in \hat{G}$ and define

$$
\begin{gathered}
B=\left\{z \in \mathbf{C}:\left(w^{\prime}, z\right) \in \hat{G}\right\}, \quad u(z)=\hat{\varphi}\left(w^{\prime}, z\right), \quad z \in B, \\
S(z)=\hat{R}\left(w^{\prime}, z\right), \quad z \in \mathbf{C}
\end{gathered}
$$

( $S$ is a complex polynomial of degree $\nu$ of the form $\hat{R}_{0} z^{\nu}+S_{1} z^{\nu-1}+\cdots+S_{\nu}$ ),

$$
\delta(z)=\delta_{\hat{G}}\left(w^{\prime}, z\right), \quad z \in \mathbf{C} .
$$

Clearly $\delta^{\mu}|S| u \leqslant c$ on $B$, so the proof reduces to the proof of the following

Lemma. Let $B$ be an open set in $\mathbf{C}$, let $u: B \rightarrow \mathbf{R}_{+}$be a subharmonic function, let $S(z)=S_{0} z^{\nu}+\cdots+S_{\nu-1} z+S_{\nu}, S_{0} \neq 0$, and let $\delta: \mathbf{C} \rightarrow[0,1]$ be such that $\delta \leqslant \delta_{B}$, $\{\delta>0\}=B,\left|\delta(z)-\delta\left(z^{\prime}\right)\right| \leqslant\left|z-z^{\prime}\right|, z, z^{\prime} \in \mathbf{C}$. Assume that $\delta^{\mu}|S| u \leqslant c$. Then

$$
\delta^{\mu+\nu} u \leqslant\left(c /\left|S_{0}\right|\right) c(\mu, \nu),
$$

where $c(\mu, \nu):=\left[2(\mu+\nu+1)(1+1 /(\mu+\nu))^{\mu+\nu}\right]^{\nu}$.

Proof of Lemma. The idea of the proof is taken from [6]. Suppose that $S(z)=S_{0}\left(z-z_{1}\right)^{\nu_{1}} \cdots\left(z-z_{r}\right)^{\nu_{r}}$, where $\nu_{1}, \ldots, \nu_{r} \in \mathbf{N}, \nu_{1}+\cdots+\nu_{r}=\nu$ and $z_{i}$ $\neq z_{j}$ for $i \neq j$. Let

$$
d(z):=\min \left\{\left|z-z_{j}\right|: j=1, \ldots, r\right\}, \quad z \in \mathbf{C} .
$$

Then

(1) $|S(z)| \geqslant\left|S_{0}\right|[d(z)]^{\nu}, z \in \mathbf{C}$.

Fix $0<\theta<\frac{1}{2}$ and define

$$
U_{\theta}=\{z \in \mathbf{C}: d(z)<\boldsymbol{\theta} \cdot \boldsymbol{\delta}(z)\} .
$$

Obviously $U_{\theta} \in$ top $C$ and $S^{-1}(0) \cap B \subset U_{\theta}$. Observe that in view of (1)

(2) $\delta^{\mu+\nu}\left(z_{0}\right) u\left(z_{0}\right) \leqslant c \theta^{-\nu} /\left|S_{0}\right|$, if $z_{0} \in G \backslash U_{\theta}$.

Similarly as in Narasimhan's proof one can obtain

(3) $U_{\theta} \Subset G$;

(4) for every connected component $U$ of $U_{\theta}$ and for every $z_{0} \in U, w_{0} \in \partial U$ : $(1-2 \theta)^{\nu} \delta\left(z_{0}\right) \leqslant \delta\left(w_{0}\right)$. 
Fix $z_{0} \in U_{\theta}$ and let $U$ denote the connected component of $U_{\theta}$ containing $z_{0}$. In view of (3), $U \Subset B$, so by the maximum principle for subharmonic functions there exists a point $w_{0} \in \partial U$ such that $u\left(w_{0}\right)=\max \{u(z): z \in U\}$. Consequently, in view of (2) and (4), we get

$$
\delta^{\mu+\nu}\left(z_{0}\right) \cdot u\left(z_{0}\right) \leqslant c /\left|S_{0}\right| \cdot\left[(1-2 \theta)^{\mu+\nu} \theta\right]^{-\nu} .
$$

Finally,

$$
\delta^{\mu+\nu} u \leqslant\left(c /\left|S_{0}\right|\right)\left[\sup \left\{(1-2 \theta)^{\mu+\nu} \theta: 0<\theta<1 / 2\right\}\right]^{-\nu}=\left(c /\left|S_{0}\right|\right) c(\mu, \nu) .
$$

Hence, Lemma 2.17 is proved.

\section{REFERENCES}

1. O. U. Balashova, Description of 2-circled $H^{x}$-domains of holomorphy, Some Problems of Multidimensional Complex Analysis, Akad. Nauk SSSR Sibirsk Otdel. Inst. Fiz., Krasnoyarsk, 1980, pp. 221-224. (Russian)

2. N. Bourbaki, Topologie générale, Hermann, Paris, 1961

3. T. W. (jamelin. Peak points for algebras on circled sets, Math. Ann. 238 (1978), 131-139.

4. J. Garnett, Positive length but zero analytic capacity, Proc. Amer. Math. Soc. 24 (1970), 696-699.

5. R. Gunning and H. Rossi, Analytic functions of several complex variables, Prentice-Hall, Englewood Cliffs, N. J., 1965.

6. R. Narasimhan, On holomorphic functions of polynomial growth in a bounded domain, Ann. Scuola Norm. Sup. Pisa 21 (1967), 161-166.

7. M. Jarnicki and P. Pflug, Non-extendable holomorphic functions of bounded growth in Reinhardt domains, Ann. Polon. Math. 46 (1985), 129-140.

8. M. Jarnicki and P. Tworzewski, A note on holomorphic functions with polynomial growth, Osnabrücker Schriften Math. 67 (1983).

9. P. Pflug, Über polynomiale Funktionen auf Holomorphiegebieten, Math. Z. 139 (1974), 133-139.

10 . , Quadratintegrale holomorphe Funktionen und die Serre-Vermutung, Math. Ann. 216 (1975), $285-288$.

11. About the Caratheodory completeness of all Reinhardt domains, Functional Analysis, Holomorphy and Approximation Theory II, North-Holland Math. Studies 86, North-Holland, Amsterdam, 1984, pp. 331-337.

12. N. Sibony, Prolongement des fonctions holomorphes bornées et métrique de Carathéodory, Invent. Math. 29 (1975), 205-230.

13. J. Siciak, Extremal plurisubharmonic functions and capacities in $\mathbf{C}^{\prime \prime}$, Sophia Kokyuroku Math. 14 (1982)

14. Circled domains of holomorphy of type $H^{\propto}$, Bull. Soc. Sci. Lett. Lodź 1 (1984/85), 1-20.

15. _ Balanced domains of holomorphy of type $H^{\infty}$, Mat. Vesnik 37 (1985), 134-144.

16. P. Tworzewski and T. Winiarski, Analytic sets with proper projections, J. Reine Angew. Math. 337 (1982), 68-76.

Uniwersytet Jagielloński, Instytut Matematyki, ul. Reymonta 4, 30 - 059 Krakow, Poland

Universität Osnabrtick, Abteilung Vechta, Driverstrasse 22, 2848 Vechta, Germany 